\title{
The prognosis in cervical epithelial changes of uncertain significance is similar to that of cervical intraepithelial neoplasia grade 1
}

\author{
M K Heatley
}

\begin{abstract}
BackgroundlAims-The term epithelial changes of uncertain significance (ECUS) describes a minor degree of nuclear pleomorphism limited to the basal layers of cervical epithelium in the absence of severe inflammation with associated normal mitoses, koilocytosis, or koilocytosis associated features. This study aimed to investigate the long term prognosis of this lesion.

Methods-Slides from 128 women with low grade cervical abnormalities, accessioned consecutively, were reviewed.

Results-In 43 women the initial diagnosis of ECUS was confirmed and in 30 women the initial diagnosis of cervical intraepithelial neoplasia grade 1 (CIN1) was confirmed. Comparison of follow up data from these 73 women revealed a similar prognosis in the two groups in terms of regression to normal, persistence of low grade disease, or progression to high grade CIN.

Conclusions-Low grade cervical disease (ECUS and CIN1) should be managed according to similar treatment protocols. (F Clin Pathol 2001;54:474-475)
\end{abstract}

Keywords: cervical intraepithelial neoplasia; diagnosis; follow up study

The term basal abnormality of uncertain significance (BAUS) was proposed by Anderson et al in $1991,{ }^{1}$ with the suggestion that it should be used if there was a minimal degree of nuclear pleomorphism limited to the basal layers of the epithelium in the absence of severe inflammation; if the features were those of cervical intraepithelial neoplasia grade 1 (CIN1), but with associated severe inflammation present immediately beneath the epithelium; or if there was thin epithelium in which a diagnosis of CIN (grade not specified) would be appropriate, which was associated with severe inflammation. The need for a category to encompass borderline lesions was re-enforced by a recent working party of the Royal College of Pathologists and the NHS cervical screening programme, ${ }^{2}$ although the term was modified to "epithelial changes of uncertain significance (ECUS)" and the diagnostic features refined to a minor degree of nuclear pleomorphism limited to the basal layers in the absence of severe inflammation, with only normal mitoses and koilocytosis or koilocytosis associated features.

Although several studies have reported poor interobserver variation for BAUS, ${ }^{34}$ perhaps reflecting a poor observer variability even in
CIN lesions, ${ }^{5}$ the importance of this lesion, in terms of its long term prognosis, has not been studied. This paper describes the long term prognosis for a group of women with low grade CIN lesions.

\section{Materials and methods}

The laboratory computer at the department of pathology, the Royal Liverpool University Hospital, was interrogated to identify women diagnosed as having low grade epithelial abnormality of the cervical squamous epithelium in the period May to August 1998. Patients with high grade lesions, invasive carcinomas, cervical polyps, and biopsies that were deemed to be inadequate were excluded from the study. In total, 128 women whose biopsies had been accessioned consecutively between May and July 1998 were identified, and the original haematoxylin and eosin sections were reviewed and assigned to the appropriate diagnostic categories. ${ }^{1}$ Follow up data were obtained from the departments of histopathology and cytopathology.

For the purposes of statistical analysis, the cytological and histological diagnoses obtained on follow up were combined into the categories no abnormality (normal biopsy or smear), borderline (borderline nuclear change on cytology or BAUS on histology), low grade (mild dyskaryosis or CIN1), high grade (moderate or severe dyskaryosis or CIN2/3), or inadequate (inadequate smear or biopsy) and are included under the heading "diagnosis on follow up" in table 1.

Women whose cervical abnormality justified follow up rather than treatment usually have a repeat colposcopy, biopsy, or cervical smear at six months and then a further procedure at 18 months. Therefore, follow up data were categorised on the basis of an initial follow up at six months and a second follow up at 18 months after the initial biopsy. The highest overall grade on follow up was also recorded.

The data were anonymised and analysed using a Statview 4.0 programme on an AppleMac performa computer. Follow up data from patients in whom a diagnosis of BAUS was made on both initial examination and at review were compared with those from patients in whom CIN1 was diagnosed on both review occasions on their initial biopsy using a $\chi^{2}$ test. A $\mathrm{p}$ value of less than 0.05 was deemed significant.

Results

One hundred and twenty eight women were identified as having a biopsy showing BAUS or CIN1. On review, the diagnosis of BAUS or 
Table 1 Frequency with which no abnormality, borderline nuclear change or basal abnormality of uncertain significance (BAUS), and dyskaryosis or cervical intraepithelial neoplasia (CIN) were diagnosed at six months and 18 months follow up and as the highest grade lesion in women in whom BAUS and CIN1 were diagnosed on an initial punch biopsy

\begin{tabular}{|c|c|c|c|c|c|c|}
\hline \multirow{2}{*}{$\begin{array}{l}\text { Diagnosis on initial } \\
\text { biopsy }\end{array}$} & \multicolumn{5}{|c|}{ Diagnosis on follow up } & \multirow[b]{2}{*}{ Total } \\
\hline & No abnormality & Borderline & Low grade & High grade & Inadequate & \\
\hline \multicolumn{7}{|l|}{6 months } \\
\hline BAUS & $14(48 \%)$ & $4(14 \%)$ & $7(24 \%)$ & $2(7 \%)$ & $2(7 \%)$ & 29 \\
\hline CIN1 & $11(41 \%)$ & $4(16 \%)$ & $6(23 \%)$ & $3(12 \%)$ & $2(8 \%)$ & 26 \\
\hline \multicolumn{7}{|l|}{18 months } \\
\hline BAUS & $15(62 \%)$ & $4(17 \%)$ & $3(12.5 \%)$ & $1(4 \%)$ & $1(4 \%)$ & 24 \\
\hline CIN1 & $11(64 \%)$ & $3(18 \%)$ & $3(18 \%)$ & 0 & 0 & 17 \\
\hline \multicolumn{7}{|c|}{ Highest overall grade on follow up } \\
\hline BAUS & $13(41 \%)$ & $8(25 \%)$ & $9(28 \%)$ & $2(6 \%)$ & 0 & 32 \\
\hline CIN1 & $11(38 \%)$ & $5(18 \%)$ & $8(29 \%)$ & $3(11 \%)$ & $1(4 \%)$ & 28 \\
\hline
\end{tabular}

CIN1 was confirmed in 73 women (43 cases of BAUS and 30 cases of CIN1).

At six months, 14 of 29 women with BAUS and 11 of 26 women with CIN1 had a normal follow up smear or biopsy (table 1). Persistent low grade or borderline disease was identified in 11 and 10 women, respectively, whereas two women with BAUS and three women with CIN1 had progressed to a high grade diagnosis. However, at 18 months most women had reverted to a normal smear or biopsy, with seven of 24 women with BAUS and six of 17 women with CIN1 having persistent low grade disease. Only one woman with an initial diagnosis of BAUS had high grade disease, reflecting the policy of promptly treating high grade cervical abnormalities. Fifty nine per cent of women with BAUS were found to have a cervical abnormality at some point during the 18 months of follow up, consisting mainly of persistent borderline lesions $(25 \%)$ or mild dyskaryosis or CIN1 $(28 \%)$. However, two patients had a high grade lesion on follow up. Fifty eight per cent of women with an initial diagnosis of CIN1 had an abnormal smear or biopsy within 18 months of follow up, comprising borderline (18\%), low grade $(29 \%)$, and high grade $(11 \%)$ lesions detected cytologically, histologically, or by both methods. One follow up smear was inadequate. The diagnoses on follow up of women with these two conditions showed no evidence of a significant difference.

\section{Discussion}

Previous studies have shown that there is considerable observer variability in histopathological reporting of low grade cervical abnormalities on biopsy specimens..$^{3-5}$ Nevertheless,
43 women were identified in whom the diagnosis both initially and after re-assessment of the slides was BAUS, and a further 30 women had a diagnosis of CIN1 on both their initial and review assessment. Comparison of follow up data from these 73 women demonstrated a similar prognosis in terms of regression to normal smear or biopsy, persistence of low grade disease, or progression to high grade CIN. The results of this small study group suggest that these two entities have a similar prognosis and they justify the subsequent colposcopic review of women in whom the diagnosis of BAUS has been made, as has been recommended previously. ${ }^{1}$ This reflects the management of CIN1 and might justify the combination of these conditions into a single low grade group. ${ }^{4}$

I am grateful to Miss K Newby for typing the manuscript, Mr G Mellor for identifying the cases, and Mr P Khanna for locating the slides.

1 Anderson ML, Brown CL, Buckley CH, et al. Current views on cervical intraepithelial neoplasia. $f$ Clin Pathol 1991;44:969-78.

2 Fox $\mathrm{H}$. Working party of the Royal College of Pathologists and the NHS cervical screening programme. Histopathology reporting in cervical screening. NHSCSP publication. No 10 reporting in cerr 1999.

3 Creagh T, Bridger JE, Kupek E, et al. Pathologist variation in reporting cervical borderline epithelial abnormalities and cervical intraepithelial neoplasia. $f$ Clin Pathol 1995;48:59-61.

4 McCluggage WG, Bharucha $\mathrm{H}$, Caughley LM, et al. Interobserver variation in the reporting of cervical colposcopic specimens: comparison of grading systems. $f$ Clin Pathol 1996;49:833-5.

5 Robertson AJ, Anderson JM, Swanson Beck J, et al. Observer variability in histopathological reporting of cervical biopsy specimens. F Clin Pathol 1989;42:231-8. 